\title{
The serial position effect in short-term memory under E- and S-paced conditions'
}

\section{NORMAN R. ELLIS AND JEANNE DUGAS, DEPARTMENT OF PSYCHOLOGY, UNIVERSITY OF ALABAMA, University, Alabama 35486}

In a STM task $S$ s were presented visually nine CVCs in a row. $A$ probe followed, requiring $S$ to remember the position of one of the CVCs. SPE were found for both $E$ - and S-paced conditions, with S-pacing facilitating primacy performance only. Latencies showed an SPE for 7 of 15 Ss. Long latencies resulted in more accurate performance in primacy, suggesting that Ss use rehearsal strategies.

The study of serial position effects (SPE) in human learning and memory has a long history and a tedious recount seems inappropriate here. No single theoretical account of the phenomenon is entirely adequate. From the viewpoint of information theory, the processes involved in learning and memory, particularly short-term memory (STM), are encoding, storage, and retrieval, and the SPE in STM could be attributed to any one or a combination of these theoretical constructs.

Most studies of SPE have presented stimulus items at a constant rate, apparently assuming that items throughout a list are encoded with equal facility. Thus, SPE in such studies are usually attributed to storage or retrieval processes.

If determinants of SPE occur in encoding, this should be reflected in item latencies, time spent viewing each item or interitem intervals in an S-paced task. On the other hand, if the SPE are due entirely to storage or retrieval processes, these measures would not show any differential effects.

\section{METHOD}

\section{Subjects and Apparatus}

The Ss were 30 undergraduates who participated to fulfill a psychology course requirement. The apparatus has been described previously (Ellis \& Hope, in press). In brief, a row of nine miniature projectors successively presented visual stimuli from left to right, and then a tenth projector located above the row "probed" memory for the position of a stimulus. That is, $\mathrm{S}$ attempted to respond to one of the positions which was identical to the probe by pressing a Plexiglas key over the position he thought was correct. The stimuli were 12 CVCs with a values ranging from .30 to .40 selected from Noble's list (Noble, 1961). All CVCs began with a different letter. A doorbell chime signaled a correct response. Interstimulus intervals, the interval between the ninth stimulus and the probe, and the interval between probe offset and response were recorded with a print-out counter and a .5-sec pulse stream generator.

Procedure

The 30 Ss were assigned equally and without bias to E-paced and S-paced groups. In both groups the duration of each CVC was .75 sec. For E-paced Ss all events occurred automatically. The offset of one stimulus was contiguous with the onset of the next, and the probe immediately followed of sset of the ninth CVC. For the S-paced condition $S$ was instructed to present stimuli to himself by pressing each position, one after the other from left to right, and then the probe. He was further instructed that he could proceed at his own pace and that this was not a "timed" task.

In both $\mathbf{E}$ - and Spaced conditions, a correction procedure was employed and $E$ manually recorded correct and incorrect responses. The $E$ preset one of 18 randomly determined sequences of nine CVCs and a probe for a trial. Each of the nine positions was probed twice in an 18-trial block, and one replication of the 18 sequences provided four "tests" for each position in the 36 trials.

RESULTS AND DISCUSSION

Figure 1 depicts per cent correct responses for E- and S-paced groups over the nine positions. An analysis of variance of these data yielded an $F(1,28)=8.7, p<.01$ for E-versus $S$-paced, $F(8,224)=15.8, p<.001$ for serial position, and $F(8,224)=3.1$, $p<.005$ for the interaction. No explanation of the coincidence of performance at Position 2 seems obvious. Figure 2 presents mean interitem latencies for the S-paced group. Figure 3 showing individual latencies in four 9-trial blocks for six selected Ss, indicates that the averaged curve in Fig. 2 is misleading. Five of these Ss show substantial changes in latency as a function of serial

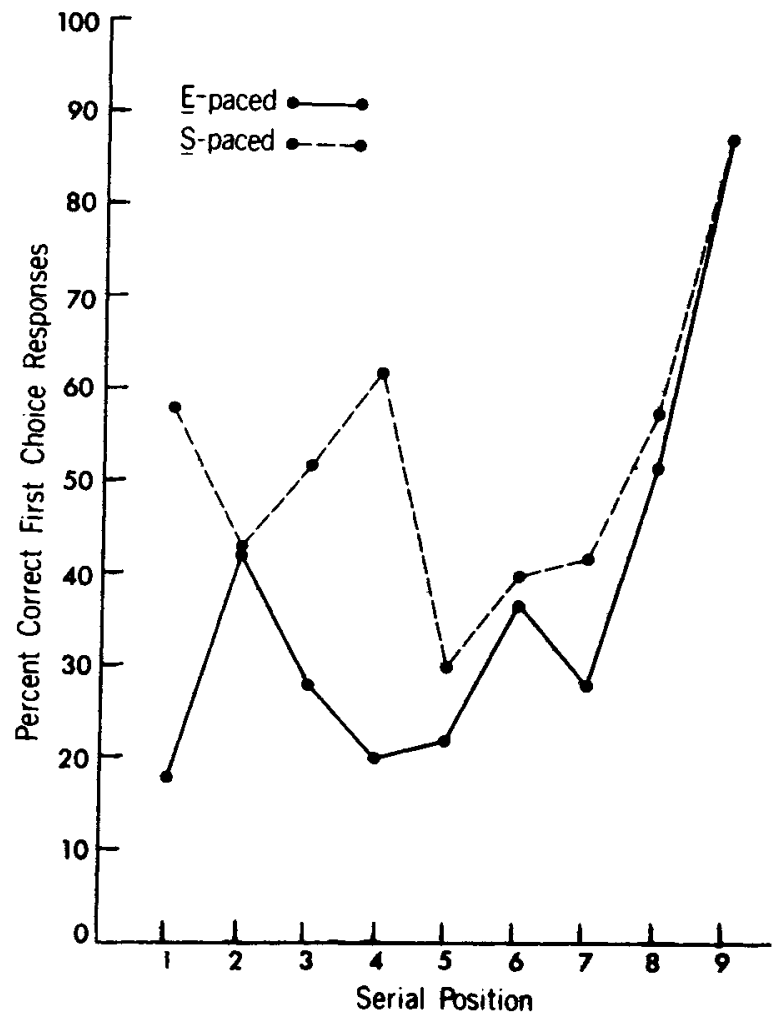

Fig. 1. Percent correct first choice responses for E- and S-paced poups at a function of serial position.

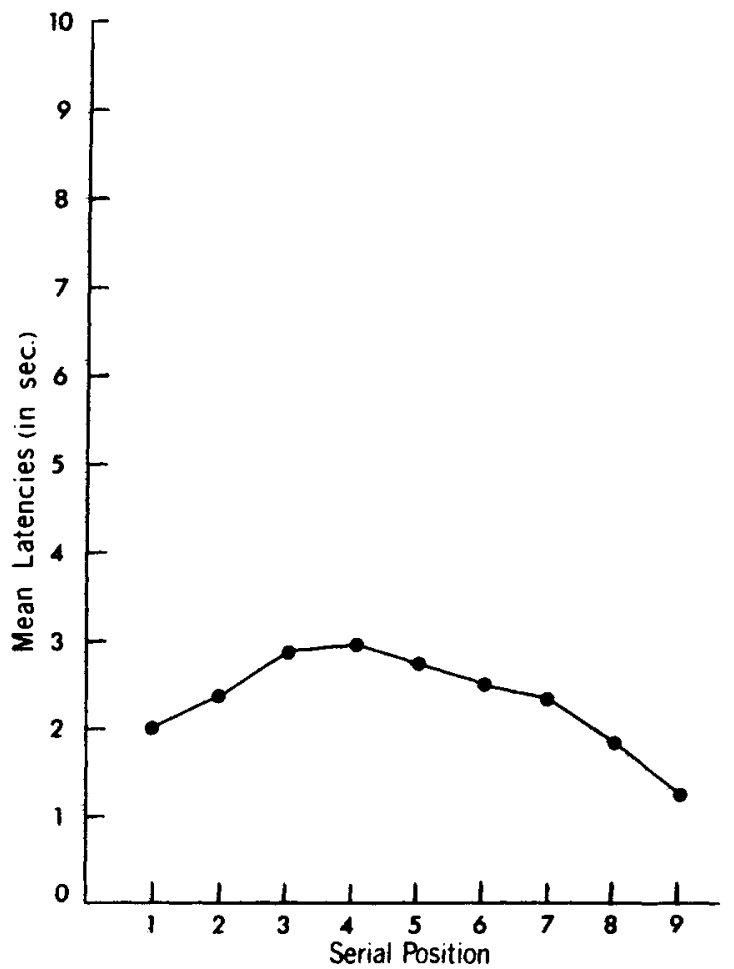

Fig. 2. Mean latencies of the S-paced group as a function of sertal poation. 

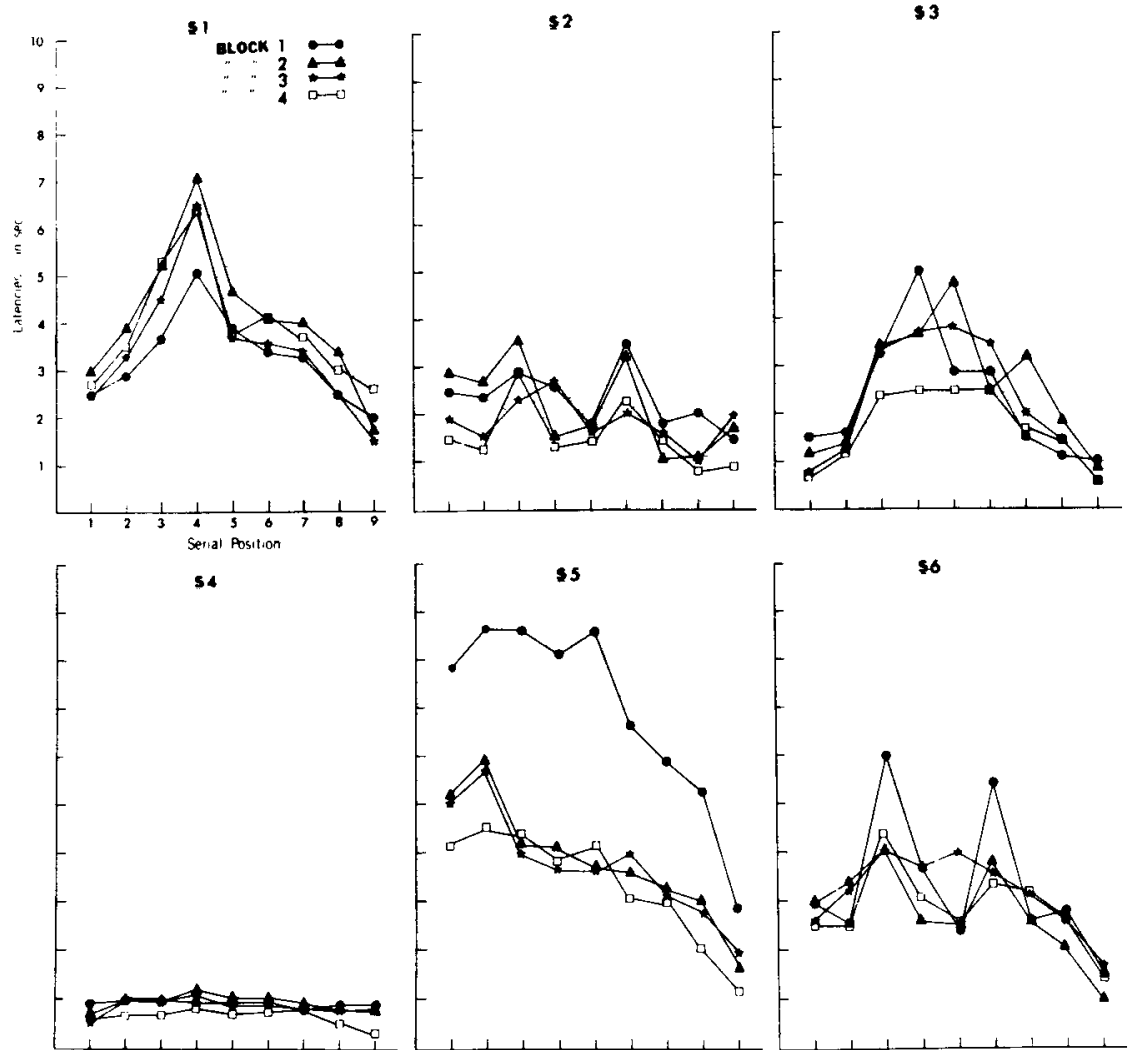

Fig. 3. Mean latencies of 6 individual Ss in the S-paced group in four 9-trial blocks. position, i.e., SPE similar to those usually found for accuracy indices. The performance of two other Ss was similar to that of these five. The performance of $S$ four is quite representative of that of seven additional Ss. For these eight Ss latencies are short,

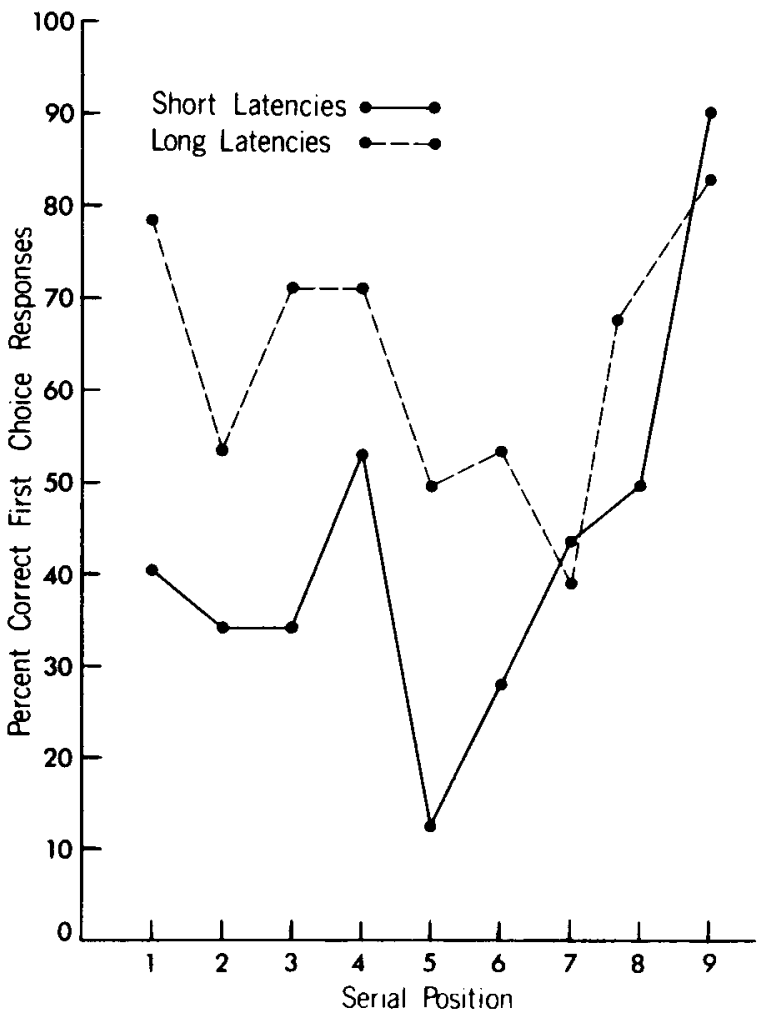

Fig. 4. Percent correct first choice responses for short-and long-latency Ss in the S-paced group. in fact, hardly more than reaction time to the offset of the preceding stimulus. Moreover, the changes over serial position are negligible.

In order to evaluate the effects of latency strategy, Fig. 4 contrasts accuracy scores of the seven Ss with long latencies with those of the eight Ss with minimal latencies. It is apparent that primacy performance is markedly facilitated for the Ss showing long interitem latencies. On the other hand, recency is invariate for these different strategies.

Figure 3 demonstrates the high intrasubject consistency which occurred in latency strategy. It was anticipated that Ss would develop a rehearsal strategy over trials which would be reflected as a changing latency pattern. This did not prove to be the case. In the main, Ss adopted a strategy initially and retained it throughout the task. Also, those Ss with minimal latencies initially continued in a similar manner over the four 9-trial blocks.

Response latencies (interval between probe and choice response) for incorrect responses were appreciably longer than those for correct responses for all $15 \mathrm{Ss}$ in the S-paced group.

These data indicate that performance in a sequentially presented STM task yields SPE which agree with previous findings (Ellis \& Hope, in press). The form of SPE is dependent upon pacing conditions with an S-pacing condition facilitating primacy performance. Recency is uninfluenced by type of pacing. Roughly half of the Ss under an S-paced procedure show an SPE in interitem latencies resembling the SPE typically found in accuracy data. Primacy performance of these Ss is substantially above that of those who do not show this latency strategy. Latency strategies appear to be highly reliable as shown by intrasubject performance curves. These findings are consistent with the hypothesis that rehearsal is an important determinant of the primacy portion of the serial position curve and are in agreement with the results of a number of studies (e.g., see Ellis \& Hope, in press) which have employed other variables within the same experimental paradigm

\section{REFERENCE}

ELLIS, N. R., \& HOPE, R. Memory processes and the serial position curve. J. exp. Psychol., in press.

\section{NOTE}

1. This research was supported by Grant MH10724 from the National Institute of Mental Health. Public Health Service. 